\title{
Classificação dos Resíduos de SERVIÇOS DE SAÚde de UM HOSPITAL VETERINÁRIO
}

\section{Classification of health SeRVices WASte from a Veterinary hospital}

\section{Rosane Regina PILGER}

Bacharel em Administração. Especialista em Gestão da Qualidade. Mestre pelo Programa de Pós Graduação em Engenharia: Energia, Ambiente e Materiais (PPGEAM-ULBRA)

\section{FLAVIA SCHENATO}

Mestre e Doutor (Instituto de Geociências-UFRGS). Professora, Doutora do Programa de Pós-Graduação em Engenharia: Energia, Ambiente e Materiais (PPGEAM-ULBRA)

\author{
Recebido: 07/02/07 Aceito: 18/12/07
}

\section{RESUMO}

O trabalho avalia aspectos referentes à classificação e segregação dos resíduos gerados no Hospital Veterinário da Ulbra (RS), a partir de estudos realizados para a elaboração do Plano de Gerenciamento dos Resíduos de Serviço de Saúde (PGRSS), para a instituição. O plano foi baseado na resolução RDC no 306/2004 da ANVISA (Brasil, 2004), que define as diretrizes para o manejo dos resíduos de instituições de saúde, incluindo a segregação, coleta, armazenamento, transporte interno e externo, tratamento e disposição final. A partir da caracterização, classificação e do diagnóstico das atividades de manejo dos resíduos do hospital, foi possível avaliar a importância da segregação, no local de origem, para a redução de resíduos que necessitam de tratamentos especiais, bem como para a redução de riscos de propagação de doenças.

PALAVRAS-CHAVES: Resíduos de serviço de saúde, classificação, segregação, hospital veterinário, Resolução no 306/2004.

\begin{abstract}
This work evaluates some referring aspects to the residues classification and segregation generated in Ulbra's Veterinary Hospital (RS), starting from studies accomplished in the elaboration of a Residues Health Service Management Plan (PGRSS), for the institution. The plan was based on RDC $n^{\circ}$ 306/2004 ANVISA's Resolution (Brasil, 2004), that defines the guidelines for handling the health institutions residues, including the segregation, collection, storage, internal and external transportation, treatment and final disposition. From the characterization, classification and the diagnostic handling activities of hospital residues, it was possible to evaluate the segregation importance, in the origin location, for the residues reduction that need special treatments, as well as for the risks reduction of diseases propagation.
\end{abstract}

KEYWORDS: Health service waste, classification, segregation, Veterinary hospital, Resolution $n^{\circ}$ 306/04.

\section{INTRODUÇÃO}

Os resíduos hospitalares, até a década de 80, estavam incluídos na classificação de resíduos perigosos conforme a norma NBR 10004/1987, da Associação Brasileira de Normas Técnicas (ABNT,1987) e Classe I perigosos, na nova versão NBR 10004/2004 (ABNT, 2004). A denominação "lixo hospitalar" foi substituída por "resíduos de serviços de saúde" com o surgimento da norma NBR 12807/1993 (ABNT, 1993), que incluí resíduos produzidos por todos os tipos de estabelecimento prestadores de serviços de saúde, tais como, hospitais, ambulatórios, consultórios médicos e odontológicos, laboratórios farmácias, clínicas veterinárias, entre outros.

No Brasil, o gerenciamento dos resíduos de serviços de saúde (RSS) foi representado através da Resolução no 05 do CONAMA, de 1993, que estabeleceu definições para a classificação e procedimentos mínimos para o gerenciamento dos resíduos de saúde. Em 2001 foi publicada a Resolução no 283 do CONAMA que veio complementar os procedimentos do gerenciamento da Resolução anterior, classificando os resíduos em 4 grupos.

Em 2003, a RDC no 33 da ANVISA modifica a classificação dos resíduos em 5 grupos. Em 2004, entra em vigor a RDC no 306, harmonizando os princípios contemplados entre CONAMA $n^{\circ} 283 / 2001$ (Brasil ,2001) e RDC n³3/2003 (Brasl, 2003). A Resolução apresenta a classificação dos resíduos em cinco grupos definido-os em: grupo A (infectantes); grupo B (químicos); grupo C (radioativos) grupo D (comum) e grupo E (perfurocortantes). Em 2005, é publicada a Resolução no 358 do CONAMA, revogando a Resolução $\mathrm{n}^{\circ} 283$, redefinindo a classificação dos resíduos de quatro para cinco grupos.

No entanto, é a RDC no 306/2004 (Brasil, 2004), atualmente em vigor, que não somente classifica e define o tratamento dos resíduos, como também define que compete ao gerador de RSS elaborar o seu Plano de Gerenciamento de Resíduos de Serviços de Saúde, que consiste em um documento que aponta e descreve as ações relativas ao manejo dos resíduos sólidos, observadas suas características e riscos, no âmbito dos estabelecimentos.

A classificação adequada dos resíduos gerados em um estabelecimento de saúde, conforme a Organização Pan-Americana de Saúde (1997) permite que seu manuseio seja eficiente, econômico e seguro. A classificação 
dos resíduos facilita uma segregação apropriada dos resíduos, reduz os riscos sanitários e gastos com seu manuseio gerando, com isso, fraçôes que exigirão medidas mais seguras e menos dispendiosas para o seu tratamento.

A heterogeneidade na composição e classificação dos resíduos merece atenção quanto ao manejo intra-unidade e ao destino dado aos mesmos, pois representam riscos ocupacionais e riscos de infecção hospitalar e ambiental, principalmente, se descartados de maneira inadequada (Salomão, Trevisan e Gunther, 2004). Para um correto gerenciamento, intra e extra-hospitalar dos resíduos de serviços de saúde, a classificação implantada deve considerar a área de geração, a natureza e o potencial de risco dos resíduos, a fim de oferecer segurança e minimizar riscos, tanto ao agente que maneja tais resíduos, quanto ao meio ambiente. $\mathrm{O}$ gerenciamento dos RSS tem como objetivo, não somente, controlar e diminuir os riscos, mas também minimizar os resíduos desde o ponto de origem. (Schneider et al, 2004).

De acordo com Garcia \& Zanetti-Ramos (2004) os princípios que devem orientar o gerenciamento dos resíduos são a redução, segregação e a reciclagem. Esses princípios devem ser incorporados ao PGRSS de todos os estabelecimentos geradores, sendo que a redução e segregação, no momento da geração, trazem economia de recursos e no tratamento dos resíduos. A legislação estabelece que, quando ocorre a mistura de resíduos comuns com perigosos, o volume total deve ser tratado como resíduo perigoso, o mesmo acontecendo para resíduos comuns quando mesclados com resíduos infectantes. Portanto, a segregação adequada, no local de geração, permite diminuir o volume de resíduos que necessitam de um manejo especial, diminuindo também os custos com o tratamento (Ribeiro Filho, 1998).

Em muitos locais, observa-se um comportamento de "tudo ou nada". Isto é, ou todos os resíduos são segregados como perigosos, ou nada é separado e, com isso, grande parte dos resíduos de serviços de saúde acaba sendo disposta como resíduos comuns ou domiciliares (Garcia \& Zanetti-Ramos, 2004). Desde que feita uma segregação adequada, parte dos resíduos de serviços de saúde poderá ser reciclada, trazendo de volta ao ciclo produtivo, materiais que seriam descartados. A segregação na origem, segundo a UCV/GTZ (1992), possibilita a reciclagem e o reuso de determinados tipos propiciando a minimização dos resíduos a serem dispostos. Akutsu e Hamada (1993) observaram que para os 17 maiores hospitais de Porto Alegre, 46,9\% dos resíduos produzidos são considerados infectantes e $53,1 \%$ são não infectantes concluindo que a porcentagem tão alta de resíduos considerados infectantes deve-se à segregação interna ineficaz do lixo. Segundo Mattos, Silva e Carrilho (1998) cerca de $20 \%$ de resíduos infectantes poderiam ser recicláveis.

Dentro deste contexto, este trabalho foi baseado em estudos realizados para a elaboração de um Plano de Gerenciamento dos Resíduos de Serviço de Saúde (PGRSS), para o Hospital Veterinário da Ulbra, de acordo com a resolução da ANVISA, RDC no 306/2004 (BRASIL, 2004). A partir do diagnóstico dos procedimentos de manejo e dos resultados da caracterização e classificação dos resíduos foi possível avaliar as operações de segregação que consistem em questóes importantes para o correto gerenciamento de resíduos de saúde.

\section{METODOLOGIA}

O Hospital Veterinário da Ulbra é um agente integrador no processo ensino-aprendizado do Curso de Medicina Veterinária, que presta serviços à comunidade com atendimento clínico, procedimentos cirúrgicos e análises de diagnóstico. Para a elaboração do Plano de Gerenciamento dos RSS do Hospital Veterinário e para uma avaliação de questôes referentes à classificaçãoe segregação dos resíduos foram realizados estudos de caracterização, tanto quantitativos como qualitativos, bem como, um diagnóstico dos procedimentos de manejo, em todos os setores do hospital, ou seja, 101 locaçôes. Estas locaçôes foram agrupadas em 4 áreas, de acordo com o tipo de serviço oferecido: Área 1 - Serviços Administrativo e Ensino (52 salas); Área 2 - Atendimento Clínico (6 salas); Área 3 - Internação e Procedimentos Cirúrgicos (26 salas) e, por fim, Área 4 - Laboratórios de Diagnóstico (17 salas).

A caracterização e classificação dos resíduos foram realizadas em etapas descritas a seguir.

\section{Coleta}

Para caracterização e classificação dos resíduos foi realizada a coleta de dados durante oito dias consecutivos (OPAS, 1997), para determinar a quantidade de geração de resíduos na origem e a identificação de sua natureza. A caracterização qualitativa consistiu na descrição da natureza dos resíduos, visando sua classificação de acordo com a RDC no 306/2004 (Brasil, 2004), enquanto que a caracterização quantitativa foi realizada a partir de pesagens diárias dos resíduos gerados dentro dos diferentes setores do hospital.

Os períodos de coleta de dados foram estabelecidos de acordo com o fluxo de atividades do hospital, com base na demanda de serviços, e na geração de resíduos dos dois últimos anos. Conforme dados fornecidos pela instituição foi possível identificar dois períodos distintos, que correspondem ao período de recesso (dezembro, janeiro e fevereiro), na qual são efetuados, basicamente, atendimentos ao público e análises laboratoriais, e período letivo, que apresenta maior fluxo de pessoas, além de procedimentos cirúrgicos, laboratoriais e atendimento do público. Assim, a primeira fase de coleta de dados foi realizada durante o mês de janeiro/2006, período de férias, e a segunda fase em abril/2006, durante o período letivo. Também ficou estabelecida que a coleta dos resíduos seria realizada uma vez ao dia, salvo se fossem necessárias mais coletas por uma questão de volume ou perigo de contaminação. O horário de coleta seguiu o mesmo horário de rotina da equipe de limpeza. Este procedimento foi aplicado em 100\% das dependências do hospital, incluindo salas administrativas, salas de aula, laboratórios, áreas de circulação, salas cirúrgicas, consultórios e salas de hospedagem.

Os resíduos foram coletados seguindo o procedimento abaixo descrito:

- Recipientes com volume de 10 a 70 litros: os sacos acondicionados nestes recipientes eram retirados diariamente, pelas funcionárias da limpeza, e substituídos por outros sacos previamente identificados com a data do próximo dia de coleta e assim sucessivamente.

- Recipientes com volume de 200 litros: os resíduos acondicionados nestes recipientes, correspondentes aos resíduos de animais das baias, foram 
recolhidos de acordo com a necessidade diária, pelos funcionários do hospital responsáveis por esta atividade. Devido ao seu grande volume e odor, normalmente eram retirados de duas a três vezes por dia e acondicionados em bombonas.

- Recipientes de papelão para acondicionamento de resíduo perfurocortante: por se tratar de material perigoso e sua geração diária muito pequena, foi estabelecido que as caixas amarelas de papelão fossem caracterizadas e pesadas em seu local de origem.

\section{Identificação}

Os sacos de acondicionamentos, brancos e pretos, de todos os setores do hospital, e as caixas amarelas de papelão, onde são armazenados os resíduos perfurocortantes, foram previamente identificados com etiquetas adesivas com o número da sala e data referente ao dia da coleta.

\section{Descrição e quantificação dos resíduos}

Após a realização de cada coleta, os resíduos foram descritos de acordo com a sua natureza e classificados conforme os grupos estabelecidos pela RDC $n^{\circ}$ 306/2004 (Brasil, 2004). Após classificação os resíduos foram pesados e os dados registrados em planilhas.

\section{RESULTADOS E DISCUSSOOES}

\section{Geração de resíduos}

A caracterização qualitativa e a classificação dos resíduos mostram a ocorrência de quatro (04) grupos (A, B, $\mathrm{D}$ e E) e a ausência de resíduos do grupo C (radioativos), em todas as áreas estudadas (Tabela 1). Entretanto, em alguns setores, frações de resíduos infectantes (A) e/ou químicos (B) encontram-se misturados com resíduos comuns (D), em recipientes destinados tanto aos resíduos infectantes (saco branco) como aos comuns (saco preto).

Nas Tabelas 2 e 3 mostram-se os resultados da geração total de resíduos e a quantidade dos resíduos separados por grupo e por área, nos dois períodos estudados. $\mathrm{Na}$ Tabela 2, observa-se que a quantidade total de resíduos produzidos no período analisado durante o mês de abril foi maior, aproximadamente, $75 \%$ em relação a janeiro, que é o período em que a demanda do Hospital diminui em função do recesso (férias). Ainda na Tabela 2, observa-se que, nos dois períodos, os resíduos do grupo A são gerados em maior quantidade, em relação aos demais tipos (B, D e E), sendo responsáveis, em média, por 74,8 a 78,6\% do total de resíduos gerados, valores acima da média de resíduos gerados em outros estabelecimentos
(Akutsu e Hamada, 1993) devido, provavelmente, à misturas com outros grupos.

Quando se consideram os resíduos gerados por áreas (Tabela 3), observa-se que a área 3 (Internação e Procedimentos Cirúrgicos) é a área que apresentou uma maior demanda de serviços, nos dois períodos analisados, com uma produção mensal de resíduos superior, em relação às demais áreas. Esta área apresentou um aumento de, aproximadamente, $84 \%$ na geração de resíduos em abril, em relação a janeiro. A área 1 (Serviços Administrativos e Ensino) e a área 4 (Laboratórios de Diagnóstico), também apresentaram um aumento significativo de $62 \%$ e $44 \%$, respectivamente, de janeiro em relação à abril, devido ao início do período letivo, enquanto que a área 2 (Atendimento Clinico) não apresentou grandes variações entre os dois períodos, pois estas atividades, independem do período de férias ou período letivo.

Através da classificação e quantificação dos resíduos foi possível constatar que o grupo A (infectante) é o mais representativo, seguido pelo grupo $\mathrm{D}$ (comum). O volume de ambos os grupos, no período letivo, é significativamente maior, em relação a janeiro, devido ao aumento de procedimentos e atividades em aulas práticas e o do número de pessoas nas dependências do Hospital. Entretanto, em todas as

Tabela I - Caracterização e classificação dos resíduos nos diferentes setores do hospital, divididos em áreas

\begin{tabular}{|c|c|c|}
\hline Área & Descrição do resíduo & Classe \\
\hline $\begin{array}{l}\text { 1- Serviços Administrativos e } \\
\text { Ensino (gabinetes, salas de aula, } \\
\text { sala de espera, quartos e cozinha de } \\
\text { residentes, farmácia, corredores e } \\
\text { banheiros) }\end{array}$ & $\begin{array}{l}\text { Papel ofício, saco plástico, lata de refrigerante, erva mate, papel } \\
\text { toalha, copo plástico, pó de café, caixa de cartão, garrafa pet, } \\
\text { dejetos de animais, papel jornal, caixas de papelāo, vidro de } \\
\text { remédios, restos de alimentos, máscaras, papel higiênico, seringas, } \\
\text { agulhas e frascos plásticos. }\end{array}$ & $\mathrm{A}, \mathrm{B}, \mathrm{D} \mathrm{E} \mathrm{E}$ \\
\hline $\begin{array}{l}\text { 2- Atendimento Clínico } \\
\text { (Consultórios) }\end{array}$ & $\begin{array}{c}\text { Gazes, ataduras, algodão, embalagens de seringa, papel toalha, luva, } \\
\text { pêlos de animais, lata de comida, copo plástico, frasco plástico, } \\
\text { agulhas, seringas e lâminas. }\end{array}$ & $\mathrm{A}, \mathrm{D}$ e $\mathrm{E}$ \\
\hline $\begin{array}{l}\text { 3- Internação e Procedimentos } \\
\text { Cirúrgicos (hospedagem e } \\
\text { tratamento clínico, salas cirúrgicas, } \\
\text { UTI, isolamento, necropsia) }\end{array}$ & $\begin{array}{l}\text { Papel toalha, luva, seringa, copo plástico, embalagens de seringa, } \\
\text { resto de comida, latas de comida, serragem, jornal, ataduras, gazes, } \\
\text { algodão, luva, agulhas, lâminas, dejetos de animais, vísceras, peças } \\
\text { anatômicas, carcaças, pêlos de animais, frasco de soro, pró-pé, } \\
\text { touca, máscara, roupa descartável, latas de refrigerante, garrafas pet } \\
\text { e sacos plásticos. }\end{array}$ & $A, D$ e $E$ \\
\hline $\begin{array}{l}\text { 4-Laboratórios de Diagnóstico } \\
\text { (Laboratórios, Raios - X e } \\
\text { Tomografia) }\end{array}$ & $\begin{array}{l}\text { Papel toalha, luva, seringa, copo plástico, embalagens de } \\
\text { seringa, gazes, algodão, agulhas, lâminas, frasco de soro, latas de } \\
\text { refrigerante, erva mate, cultura gelatinosa, papel kraft, espátula } \\
\text { de madeira, frasco de solvente, frasco produtos químicos, potes } \\
\text { plásticos, jornal, papel ofício, revelador e fixador. }\end{array}$ & $A, B, D$ e $E$ \\
\hline
\end{tabular}


áreas estudadas, foram constatadas uma segregação inadequada e misturas de grupos de resíduos, que será discutido a seguir.

\section{Segregação}

A caracterização qualitativa e a classificação dos RSS do Hospital permitiram constatar que os resíduos, nem sempre, são segregados adequadamente, na origem. Considerando as quatro áreas estudadas foram observados resíduos infectantes (grupo A) misturados com comuns (grupo D), em recipientes designados para resíduos comuns (sacos pretos) ou, ao contrário, resíduos comuns e/ou químicos (grupo B) misturados com infectantes, em recipientes designados para resíduos infectantes (sacos brancos). Nas Figuras 1 e 2 mostram-se os percentuais de resíduos misturados, acondicionados em sacos pretos e sacos brancos.

Com a finalidade específica de estimar o percentual dos diferentes grupos de resíduos que se encontravam misturados, nas quatro áreas, foi realizada uma análise quantitativa durante
Tabela 2- Total de resíduos produzidos durante oito (08) dias, em janeiro e abril de 2006

\begin{tabular}{ccccc}
\hline & \multicolumn{2}{c}{$\begin{array}{c}\text { Janeiro/2006 } \\
\text { (período de férias) }\end{array}$} & \multicolumn{2}{c}{$\begin{array}{c}\text { Abril/2006 } \\
\text { (período letivo) }\end{array}$} \\
Grupos & Peso (kg) & $\%$ & Peso (kg) & $\%$ \\
\hline Grupo A & 535,6 & 74,8 & 987,3 & 78,6 \\
Grupo B & 14 & 2,0 & 14 & 1,1 \\
Grupo D & 144,2 & 20,1 & 229,4 & 18,3 \\
Grupo E & 22,2 & 3,1 & 25,4 & 2,0 \\
Total de & 716,0 & 100,0 & $1.256,0$ & 100,0 \\
resíduos & & & & \\
\hline
\end{tabular}

Tabela 3 - Total de resíduos separados por área, produzidos durante oito (08) dias, em janeiro e abril de 2006

\begin{tabular}{ccccc}
\hline & \multicolumn{2}{c}{$\begin{array}{c}\text { Janeiro/2006 } \\
\text { (período de férias) }\end{array}$} & \multicolumn{2}{c}{$\begin{array}{c}\text { Abril/2006 } \\
\text { (período letivo) }\end{array}$} \\
Áreas & Peso $(\mathrm{kg})$ & $\%$ & Peso $(\mathrm{kg})$ & $\%$ \\
\hline Área 1 & 140,9 & 19,7 & 228,6 & 18,2 \\
Área 2 & 13,7 & 1,9 & 14,3 & 1,1 \\
Área 3 & 520,0 & 72,6 & 953,4 & 75,9 \\
Área 4 & 41,4 & 5,8 & 59,7 & 4,8 \\
Total de & 716,0 & 100,0 & $1.256,0$ & 100,0 \\
resíduos & & & & \\
\hline
\end{tabular}

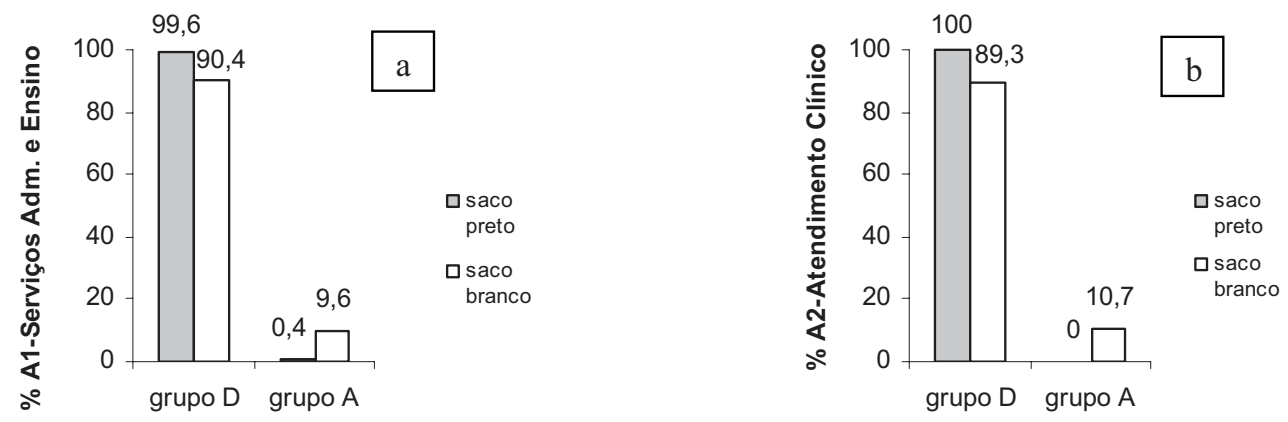

Figura I - (a) Percentual de resíduos dos grupos A e D misturados e acondicionados em sacos preto e branco, na área I. (b) Percentual de resíduos dos grupos A e D misturados e acondicionados em sacos preto e branco, na área 2
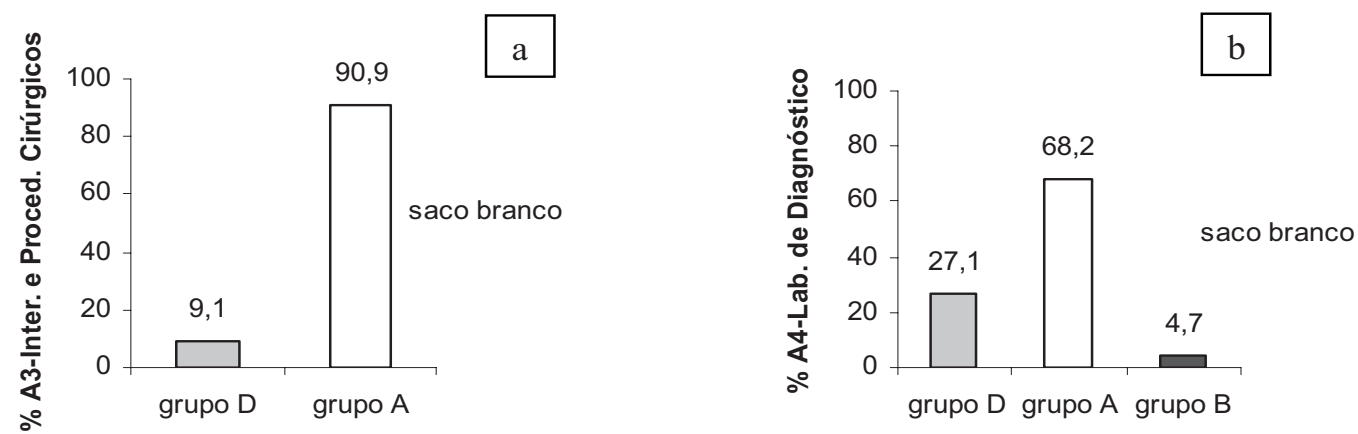

Figura 2 - (a) Percentual de resíduos dos grupos A e D misturados e acondicionados em sacos brancos, na área 3. (b) Percentual de resíduos dos grupos A, B e D misturados e acondicionados em saco branco, na área 4 
um período de coleta de 24 horas. A Tabela 4 descreve quantitativamente os resíduos, por grupo, misturados nos dois tipos de acondicionamento (sacos pretos e brancos).

De acordo com a análise quantitativa (Tabela 4), 19,3\% de resíduos comuns (grupo D) são encaminhados para tratamento e disposição como resíduos infectantes (grupo A). Isto representa uma fração considerável do total de resíduos que estão sendo tratados desnecessariamente, com autoclavagem e incineração, e encaminhados ao aterro industrial. Tal quantidade poderia ser menor, caso houvesse a segregação dos RSS em sua origem.

A Tabela 5 mostra uma projeção da quantidade de resíduos que são encaminhados desnecessariamente para tratamento e disposição final, a partir de um balanço que utiliza à quantidade média de resíduos gerados, nos dois períodos, e a percentagem de resíduos do grupo D misturados com os do grupo A, conforme observado na Tabela 4. Os resultados mostram quantidades, em torno de $7.594 \mathrm{~kg} / \mathrm{ano}$, de resíduos do grupo $\mathrm{D}$ misturados com os do grupo A. Com a segregação adequada poderia haver economia de custos no tratamento e disposição destes resíduos (Salomão, Trevisan e Gunther 2004, Garcia e Zanetti-Ramos 2004, Ribeiro Filho, 1998) e a reciclagem dos resíduos comuns (Mattos, Silva e Carrilho, 1998).

A Tabela 6 mostra que a quantidade anual de resíduos infectantes (A), misturados com os resíduos comuns (D) é, em torno, de $65,6 \mathrm{~kg}$, que corresponde a um percentual de $0,7 \%$ do total de resíduos misturados (Tabela 3). Esta quantidade, embora não muito alta, é encaminhada, atualmente, ao aterro sanitário expondo, aos que manipulam estes resíduos e ao meio ambiente, o risco de uma possível contaminação.

\section{CONCLUSÕES}

$\mathrm{Na}$ avaliação dos procedimentos atuais de manejo dos RSS do Hospital Veterinário foi possível constatar que os resíduos não são segregados adequadamente, na origem. Em geral, observa-se que resíduos comuns do grupo $\mathrm{D}$ ocorrem misturados com resíduos infectantes do grupo A. A segregação dos diferentes grupos de resíduos é importante para impedir que pequenas

Tabela 4 - Mistura de grupos de resíduos nas quatro áreas

\begin{tabular}{|c|c|c|c|c|c|}
\hline & \multicolumn{3}{|c|}{$\begin{array}{c}\text { Saco branco } \\
\text { (resíduos Infectantes) }(\mathrm{kg})\end{array}$} & \multicolumn{2}{|c|}{$\begin{array}{l}\text { Saco preto (resíduos } \\
\text { Comuns) }(\mathrm{kg})\end{array}$} \\
\hline & Grupo A & Grupo B & Grupo D & Grupo A & Grupo D \\
\hline Área 1 & 0,3 & - & 2,8 & 0,1 & 14 \\
\hline Área 2 & 2,5 & - & 0,4 & - & 0,1 \\
\hline Área 3 & 29 & - & 2,9 & - & - \\
\hline Área 4 & 7,3 & 0,5 & 2,9 & & \\
\hline Sub-total & 37,1 & 0,5 & 9 & 0,1 & 14,1 \\
\hline Total & & \multicolumn{2}{|c|}{46,6} & \multicolumn{2}{|c|}{14,2} \\
\hline$\%$ & 79,6 & 1,1 & 19,3 & 0,7 & 99,3 \\
\hline
\end{tabular}

Tabela 5 - Total de resíduos do grupo D misturados com grupo A e a estimativa anual de resíduos comum tratados

\begin{tabular}{lccc}
\hline Grupo A & $\begin{array}{c}\text { Resíduo de } \\
8 \text { dias } \\
(\mathrm{kg})\end{array}$ & $\begin{array}{c}\text { Total do } \\
\text { resíduo } \\
(\mathrm{kg}) \text { anual }\end{array}$ & $\begin{array}{c}19,3 \% \text { do grupo D } \\
\text { misturado com A } \\
(\mathrm{kg})\end{array}$ \\
\hline Período de férias (3 meses) & 535,6 & $6.025,5$ & 1.163 \\
Período letivo (9 meses) & 987,3 & $33.321,6$ & 6.431 \\
Total & & $39.347,2$ & 7.594 \\
\hline
\end{tabular}

Tabela 6 - Total dos resíduos do grupo A misturados no grupo D e a estimativa anual de resíduos infectantes não tratados

\begin{tabular}{cccc}
\hline Grupo D & $\begin{array}{c}\text { Resíduo de 8 } \\
\text { dias (kg) }\end{array}$ & $\begin{array}{c}\text { Total do } \\
\text { resíduo } \\
(\mathrm{kg}) \text { anual }\end{array}$ & $\begin{array}{c}\text { 0,7\% do grupo A } \\
\text { misturado com D } \\
(\mathrm{kg})\end{array}$ \\
\hline Período de férias (3 meses) & 144,2 & $1.623,4$ & 11,4 \\
Período letivo (9 meses) & 229,4 & $7.742,7$ & 54,2 \\
Total & & $9.366,1$ & 65,6 \\
\hline
\end{tabular}

frações de resíduos infectantes ou químicos contaminem a totalidade. A legislação estabelece que, quando resíduos infectantes são misturados aos comuns, todo resíduo deve ser tratado como infectante, inviabilizando, muitas vezes, técnica ou financeiramente um sistema adequado. Os resultados sugerem que, com a segregação adequada, poderia haver uma redução da quantidade e de custos para o tratamento e disposição dos resíduos infectantes e um maior aproveitamento dos resíduos comuns para a reciclagem.

De acordo com este estudo, um dos fatores que contribui para segregação deficiente é o fato das áreas estarem desprovidas de recipientes adequados em tamanho, tipo e identificação para a correta separação. Ainda, o treinamento adequado dos funcionários responsáveis pela coleta dos resíduos e programas de conscientização implementados pelo Hospital poderia diminuir a probabilidade de mistura nas operaçôes de manejo intra-hospitalar.

\section{AGRADECIMENTOS}

Os autores agradecem à Direção do Hospital Veterinário da Ulbra pelo apoio logístico para o desenvolvimento do trabalho.

\section{REFERÊNCIAS}

ABNT-ASSOCIAÇÃO BRASILEIRA DE NORMAS TECNICAS. NBR 10.004: Resíduos Sólidos: classificação. São Paulo, 1987.

ABNT-ASSOCIAÇÃO BRASILEIRA DE NORMAS TÉCNICAS. NBR 12.807: Resíduo de Serviços de Saúde: terminologia. São Paulo, 1993.

ABNT-ASSOCIAÇÃO BRASILEIRA DE NORMAS TÉCNICAS. NBR 10.004: Resíduos Sólidos: classificação. São Paulo, 2004. 
AKUTSU, J. \& HAMADA, J. Resíduos de Serviços de Saúde: Avaliação de Aspectos Quali-Quantitativos. In: I SEMINÁRIO INTERNACIONAL SOBRE RESÍDUOS SÓLIDOS HOSPITALARES, Cascavel, PR, 1993.

BRASIL. Agência Nacional de Vigilância Sanitária. Resolução RDC no 33, de 25 de fevereiro de 2003. Dispóe sobre o Regulamento Técnico para o gerenciamento de residuos de serviço de saúde. Diário Oficial [da] República Federativa do Brasil, Brasília, DF, mar. 2003.

Agência Nacional de Vigilância Sanitária. Resolução $R D C n^{\circ} 306$, de 07 de dezembro de 2004. Dispõe sobre o Regulamento Técnico para o gerenciamento de resíduos de serviço de saúde. Diário Oficial [da] República Federativa do Brasil, Brasília, DF, dez. 2004.

Conselho Nacional do Meio Ambiente. Resolucão $n^{\circ}$ 05, de 5 de agosto de 1993. Define as normas minimas para tratamento de resíduos sólidos oriundos de serviços de saúde, portos e aeroportos e terminais rodoviários e ferroviários. Diário Oficial [da] República Federativa do Brasil, Brasília, DF, ago. 1993.

Conselho Nacional do Meio Ambiente. Resolução no 283, de 12 de julho de 2001. Dispóe sobre o tratamento e destinação final dos residuos de serviço de saúde. Diário Oficial [da] República Federativa do Brasil, Brasília, DF, out. 2001.

Conselho Nacional do Meio Ambiente. Resolução no 358, de 29 de abril de 2005. Dispõe sobre o tratamento e disposição final dos resíduos de serviço de saúde e dá outras providências. Diário Oficial [da] República Federativa do Brasil, Brasília, DF, abr. 2005.

MATTOS, E. D; SILVA, S. A.; CARRILHO, C. M. D. M. Lixo reciclável: uma experiência aplicada no ambiente hospitalar. In: VI CONGRESSO
BRASILEIRO DE CONTROLE DE INFECÇÃO E EPIDEMIOLOGIA HOSPITALAR, Campos do Jordão. Resumos. São Paulo: ABIH, p. 1920. 1998 .

ORGANIZAÇÃO PAN-AMERICANA DE SAÚDE (OPAS) - CENTRO PAN-AMERICANO DE ENGENHARIA SANITÁRIA E AMBIENTAL. Guia para o Manejo Interno de Resíduos Sólidos em Estabelecimentos de Saúde. Tradução de Carol Castillo Argüello. Brasília (DF). Organização Pan-Americana da Saúde (OPAS), 1997.

GARCIA, L.P.; ZANETTI-RAMOS, B.G. Gerenciamento dos resíduos de serviços de saúde: uma questão de biossegurança. Cad. Saúde Pública. [online]. 2004, vol. 20, no. 3 [citado 2007-01-18], pp. 744-752. Disponível em: <http://www.scielosp. org/scielo.php?script=sci arttext\&pid=S0102 $311 \mathrm{X} 2004000300011 \& \operatorname{lng}=\mathrm{pt} \& \mathrm{nrm}=$ iso $>$. ISSN 0102-311X. Acessoem: 15 set. 2006.

RIBEIRO FILHO, O. V. Aspectos sanitários e ambientais apresentados pelos residuos de serviços de saúde. In: ASSOCIAÇĀO BRASILEIRA DE LIMPEZA PÚBLICA, São Paulo, SP. Gerenciamento dos resíduos de serviços de saúde, 1998.

SALOMÃO, I.S., TREVIZAN, S.D.P., GUNTHER, W.M.R. Segregação de Resíduos de Serviços de saúde em Centros Cirúrgicos. Engenharia Sanitária e Ambiental 111, v. 9, n. 2, p. 108-111, abr/jun 2004.

SCHNEIDER, V. E. et al. Manual de Gerenciamento de Resíduos Sólidos em Serviços de Saúde. 2 ed. Caxias do Sul, Ed. EDUCS, 319 p, 2004.

UCV/GTZ. Classificacion y cuantificacion de los desechos generados en los centros hospitalarios de $\mathrm{Ca}$ racas. In: III SEMINÁRIO INTERNACIONAL SOBRE RESIDUOS SÓLIDOS HOSPITALARES, Anais, Cascavel-PR. 1993.
Endereço para correspondência:

Rosane Regina Pilger

Programa de Pós-Graduação em

Engenharia: Energia, Ambiente e

Materiais (PPGEAM-ULBRA)

Av. Farroupilha, 800 I- Prédio 29,

Sala 230

Bairro São José

92450-900 Canoas - RS - Brasil

Tel.: (5I) 3477-9285

rrpilger@hotmail.com

flavia.schenato@ulbra.br 